\title{
AUTOMATED EXTRACTION OF BLOOD VESSEL NETWORKS FROM 3D MICROSCOPY IMAGE STACKS VIA MULTI-SCALE PRINCIPAL CURVE TRACING
}

\author{
Erhan Bas, Nastaran Ghadarghadar, Deniz Erdogmus \\ Cognitive Systems Laboratory, Northeastern University, Boston, MA, USA
}

\begin{abstract}
Blood vessel segmentation, that is, extraction of the center lines and corresponding local cylinder radii are important for the study of vascular diseases, and in the brain also important for the modeling and understanding of relationships between hemodynamics and electrical neural activity. Several image processing methods have been proposed for vessel extraction in many domains including those that explore the use of pattern recognition techniques, model-based approaches, tracking based approaches, artificial based approaches, neural network based approaches, and miscellaneous tube-like object detection approaches. In this paper, we propose a ridge tracing approach based on recently developed principal curve (PC) projection and tracing algorithms for the extraction of vasculature networks in the brain from 3D microscopy image stacks. Results on mice brain imagery obtained for the purpose of studying hemodynamic effects on neural activity are promising.
\end{abstract}

Keywords - Vessel tracing, 3D tube extraction, principal curves, bifurcation detection

\section{INTRODUCTION}

Acquisition and storage of 3D microscopy imagery for biological tubular networks such as neurons and blood vessels is widespread in basic and clinical neuroscience. Structures of neural arbors are investigated to better understand the network formation strategies in the brain and elsewhere in the nervous system. Analysis of blood vessel networks in the brain is particularly important in improving our models and understanding of the relationships between hemodynamic parameters and neural function and electrical activity. A study of vasculature networks in other contexts such as the study of stroke or trauma is also important.

Several methods have been proposed for vessel tracing and extraction in 3D imagery. These algorithms range from pattern recognition techniques to tracking-based approaches. An overview of different techniques on blood vessel extraction is given by [1].

This work is supported by NSF under grants ECCS0929576, ECCS0934506, IIS0934509, IIS0914808, and BCS1027724. The opinions presented here are solely those of the authors and do not necessarily reflect the opinions of the funding agency.
Two main methods that extract the blood vessel centerlines, also the focus of this paper and the proposed approach, are skeleton- and tracking-based approaches. Skeleton-based approaches apply local operators to the whole image. Generally, they follow these steps: 1) apply thresholds to features; 2) determine object connectivity; 3) apply thresholds again; 4) perform a suitable thinning procedure; 5) extract the skeleton in the form of a graph. By connecting the centerlines a vessel tree/graph is constructed. The resulting network structure is used for 3D reconstruction; skeleton-based approaches have been primarily applied on 3D CT images [2]. The skeleton models provide bifurcation point information besides the centerlines; however, they do not directly provide radius/boundary information. On the other hand, trackingbased approaches typically start from an initial point and detect vessel centerlines or boundaries by analyzing the pixels orthogonal to the tracking (tracing) direction. Different methods are employed in determining vessel contours or centerlines. Edge detection operation followed by sequential tracing by incorporating connectivity information is a straightforward approach [3-6]. Some tracking approaches utilize a model in the tracking process and incrementally segment the vessels [7]. A more sophisticated approach is to use a graph representation. The segmentation process is then reduced to finding the optimum path in a graph representation of the image [8]. Vessel tracking approaches can allow user intervention in initializing (seed point entry by user) as well as during tracing (path correction by user). Such manual interventions, however, are in general undesirable for large scale data analysis scenarios since it is infeasible to require too much human interaction time and effort.

In this paper we demonstrate a recursive principal curve (PC) ridge tracing approach to automatic vessel extraction in 3D imagery. Specifically, we demonstrate the application of the technique in extracting the blood vasculature networks in a section of the mice brain using 3D microscopy image stacks. The method seamlessly incorporates tube radius estimates from an isotropic Gaussian kernel Frangi filter [6] with the principal curve tracing framework using the common underlying kernel smoothing interpolation model for intensities. Simultaneous noise reduction, background elimination, and vessel enhancement is achieved with preprocessing, making the centerline identification and tracking task easier and more reliable. A multiscale 
interpolation is used in conjunction with a principal curve tracing algorithm that performs repeated propagationcorrection updates gives the vessel centerlines. The same framework also allows us to detect bifurcation points and trace branches automatically in a recursive fashion.

\section{METHODS}

The proposed approach consists of three major steps: (1) the Frangi filter is applied to the grayscale 3D imagery using isotropic Gaussian kernels (other choices obviously possible), which yields as output vesselness measures for each voxel as well as maximum vesselness response kernel bandwidth; (2) using the maximum response kernel bandwidth, a multiscale local kernel interpolation of intensity is obtained and an algorithm for projecting voxels onto their respective principal curves (ridges) with a hill climbing procedure is employed; (3) using a seed point (also projected onto the principal curve) the principal curve is traced and branches and bifurcations are determined from the samples projected onto the principal curve locally in step two. When all existing branches are traced, a network/graph of vessel centerlines and estimated radii for each sample is obtained.

\subsection{Frangi-Filter}

We apply the Frangi filter to 3D microcopy image stacks containing blood vessel images as a pre-processing step to enhance the edges and reduce noise [5]. Frangi-filter uses conditions and measures obtained from the eigenvalues of the Hessian of the interpolated image intensity to estimate the vesselness measure, using interpolations at multiple scales $(\sigma)$ since local tube radii are unknown and must be discovered. The maximum filter response is obtained within the range of vessel widths for each voxel. The filter response is large along the centerlines of the tubular structures, low towards the boundary and close to zero outside tubular regions. In addition to filtered images, Frangi-filter returns a matrix with the scales (kernel bandwidth) at which the maximum response is found for each voxel. Along the centerline, maximum response scale value is equal to the local tube radius and its value increases as the distance from the centerline increases.

These bandwidths can be used in constructing an isotropic variable bandwidth (multiscale) Gaussian kernel interpolation of the image intensity over space for the principal curve analysis to be performed in the next step.

\subsection{Principal Curve Analysis}

Let $\mathbf{p} \in \mathbb{R}^{n}$ be a position vector and let $\mathbf{p}_{1}, \mathbf{p}_{2}, \ldots, \mathbf{p}_{N}$, be a set of voxel positions in an image. Let $f(\mathbf{p})$ be the intensity function over space at position $\mathbf{p}$ and let $\mathbf{g}(\mathbf{p})$ be the transpose of its gradient vector and $\mathbf{H}(\mathbf{p})$ be the Hessian matrix. Similarly defining the Hessian of $\log f(\mathbf{p})$ and considering its second order Taylor series approximation, we define a local covariance inverse $\mathbf{F}(\mathbf{p})=-\boldsymbol{H}_{\log f}(\mathbf{p})=$ $-f(\mathbf{p})^{-1} \mathbf{H}(\mathbf{p})+f(\mathbf{p})^{-2} \mathbf{g}(\mathbf{p})^{T} \mathbf{g}(\mathbf{p})$ in anology with the covariance of a Gaussian distribution. Let $\left\{\left(\lambda_{1}(\mathbf{p}) ; \mathbf{q}_{1}(\mathbf{p})\right), \ldots,\left(\lambda_{n}(\mathbf{p}) ; \mathbf{q}_{n}(\mathbf{p})\right)\right\}$ be the eigenvalueeigenvector pairs of $\mathbf{F}(\mathbf{p})$, sorted in ascending order: $\lambda_{1} \leq \lambda_{2} \leq \cdots \leq \lambda_{n}$. In general, a point $\mathbf{p}$ is on the $d$ dimensional principal manifold if and only if the local gradient is a linear combination of eigenvectors of the local covariance inverse that span the tangent space; hence the gradient is also orthogonal to the remaining $n$ - $d$ eigenvectors for which all corresponding eigenvalues are strictly positive [9]. For instance, let $\mathbf{S}_{\perp}(\mathbf{p})=\operatorname{span}\left\{\mathbf{q}_{d+1}(\mathbf{p})\right.$, $\left.\mathbf{q}_{d+2}(\mathbf{p}), \ldots, \mathbf{q}_{n}(\mathbf{p})\right\}$ be the normal space spanned by $n-d$ eigenvectors and $S_{\|}(\mathbf{p})=\operatorname{span}\left\{\mathbf{q}_{1}(\mathbf{p}), \ldots, \mathbf{q}_{d}(\mathbf{p})\right\}$ be the tangent space spanned by $d$ vectors at $\mathbf{p}$. The condition that for $\mathbf{p}$ in the $d$-dimensional principal surface $\mathbf{g}(\mathbf{p}) \in S_{\|}(\mathbf{p})$ and the eigenvalues for the vectors that $\operatorname{span} S_{\perp}(\mathbf{p})$ are all positive reduces to the following for 1-dimensional principal curves: the gradient is an eigenvector of the Hessian and the eigenvalues for the other eigenvectors are all positive. The eigenvalue corresponding to the gradient can be negative or positive; if positive, then it is the smallest such eigenvalue in accordance with Frangi's intuition. By construction, the local maxima of the function as well as some saddle points are members of the principal curve. Consequently, given a seed point that is inside a vessel, a gradient ascent to the nearest local maximum provides a corrected seed point which is on the principal curve - the vessel centerline. Starting from such a corrected seed point, one can trace the centerline by following the tangent space eigenvector of $\mathbf{F}$ by locally identifying its smallest eigenvector due to the reasoning provided above. Since a step in the tangent vector direction (corresponding to an Euler integration of the vector flow field) will cause the trajectory to diverge from the principal curve, one can improve each iterate by performing one or more correction steps where the candidate point is iterated towards the principal curve using the projection of the local gradient on the orthogonal space $\mathbf{S}_{\perp}(\mathbf{p})$. A measure of how close the point $\mathbf{p}$ is to the principal curve, which works well once sufficiently close to the principal curve, is the cosine of the angle between the gradient and Hessian times the gradient; when on the principal curve, since these vectors are aligned, the cosine in question assumes value of unity. Specifically, we measure the cosine of this angle as follows:

$$
\gamma(\mathbf{p})=\frac{\mathbf{g}(\mathbf{p})^{T} \mathbf{F}_{\perp}(\mathbf{p}) \mathbf{g}(\mathbf{p})}{\|\mathbf{F}(\mathbf{p}) \mathbf{g}(\mathbf{p})\|\|\mathbf{g}(\mathbf{p})\|}
$$

where $\mathbf{Q}_{\perp}=\left[\mathbf{q}_{d+1}(\mathbf{p}), \mathbf{q}_{d+2}(\mathbf{p}), \ldots, \mathbf{q}_{n}(\mathbf{p})\right]$ and $\boldsymbol{\Lambda}_{\perp}=$ $\operatorname{diag}\left(\lambda_{d+1}, \ldots, \lambda_{n}\right)$ are eigenvectors that span $\mathbf{S}_{\perp}$ and their eigenvalues, respectively. Also, $\mathbf{F}_{\perp}(\mathbf{p})=\mathbf{Q}_{\perp} \boldsymbol{\Lambda}_{\perp} \mathbf{Q}_{\perp}^{T}$. Clearly, $\gamma(\mathbf{p})$ attains a value of 0 on the principal curve and is bounded from below and above by -1 and 1 , respectively. 
To obtain a smooth but local-scale-dependent interpolation for $f(\mathbf{p})$, we employ variable-width local Gaussian kernel smoothing defined as follows:

$$
f(\mathbf{p})=\sum_{i=1}^{N} w\left(\mathbf{p}_{i}\right) G_{\mathbf{\Sigma}_{i}}\left(\mathbf{p}-\mathbf{p}_{i}\right)
$$

where $w\left(\mathbf{p}_{i}\right)$ is the intensity at pixel with coordinate $\mathbf{p}_{i}$ and $\boldsymbol{\Sigma}_{i}=\sigma_{i}^{2} \mathbf{I}$ is the isotropic variable kernel covariance which is the scale at which the maximal Frangi filter output for this pixel was achieved. The Gaussian kernel is given by $G_{\boldsymbol{\Sigma}_{i}}(\mathbf{z})=C_{\boldsymbol{\Sigma}_{i}} e^{-\frac{1}{2} \mathbf{z}^{T} \boldsymbol{\Sigma}_{i}^{-1} \mathbf{z}}$. Letting $c_{i}(\mathbf{p})=w\left(\mathbf{p}_{i}\right) G_{\boldsymbol{\Sigma}_{i}}\left(\mathbf{p}-\mathbf{p}_{i}\right)$ and $\mathbf{u}_{i}(\mathbf{p})=\boldsymbol{\Sigma}_{i}{ }^{-1}\left(\mathbf{p}-\mathbf{p}_{i}\right)$, we get the gradient and Hessian as follows:

$$
\begin{aligned}
& \mathbf{g}(\mathbf{p})=-\sum_{i=1}^{N} c_{i}(\mathbf{p}) \mathbf{u}_{i}(\mathbf{p}) \\
& \mathbf{H}(\mathbf{p})=\sum_{i=1}^{N} c_{i}(\mathbf{p})\left(\mathbf{u}_{i}(\mathbf{p}) \mathbf{u}_{i}^{T}(\mathbf{p})-\mathbf{\Sigma}_{i}^{-1}\right)
\end{aligned}
$$

Using a variable kernel bandwidth allows the model to capture local scales of vessels in the interpolation process and leads to more accurate details overall compared to smoothing using a fixed bandwidth kernel.

In a scenario, where multiple principal curves intersect, local tracing methods make decisions based on the local evidence, i.e. particle analysis and edge information. Here, we propose a method that uses the described tracing strategy for fractal analysis as well. After each termination of the tracing, we employ a local search algorithm in the vicinity of the traced branch to find a new seed candidate. We constrain our search space to the underlying principal curves of the fractals. For that purpose, we calculated the projection of sample points around the underlying principal curves before starting tracing process. Similar to the tracing, Euler integration is used to solve ordinary differential equation at an arbitrary point $\mathbf{p}$ [9] and using the $\mathbf{Q}_{\perp}$ data samples are projected to their corresponding principal curves. We only process and keep the samples that are in the vicinity of the principal curve, such that if the curve length during the projection is larger than 0.5 we discard those samples, where 0.5 is the half width of a voxel. Fig. 1(a) shows such a case, where initial tree-like structure is overlaid with its principal curve projections represented with green dots. In Fig.1(b) red curve represents the initial tracing result and yellow cross denotes a seed point where tracing initiated. After each tracing process, we define proximity for the traced branch. We define this proximity as the encapsulating curvilinear cylinder envelop having inner radius $1.5 * \mathrm{R}(\mathbf{p})$, and outer radius $3^{*} \mathrm{R}(\mathbf{p})$, where $\mathrm{R}(\mathbf{p})$ is the local radius estimate of the Frangi filter. Selection of the outer radius is arbitrary but the inner radius should be larger than $\mathrm{R}(\mathbf{p})$ due to the multiscale analysis and the measure defined in (2). The purpose is to prevent the gap between underlying principal curves resulting from local eigen-value ranking system. Since this discussion is not in the scope of this paper and due to the space limitations, we left the detailed explanation of the possible gap between principal curves around bifurcation location in a future work.

In order to analyze bifurcations we cluster the principal curve samples that are nearby of the branch being traced at the previous iteration. Samples in the curvilinear cylinder
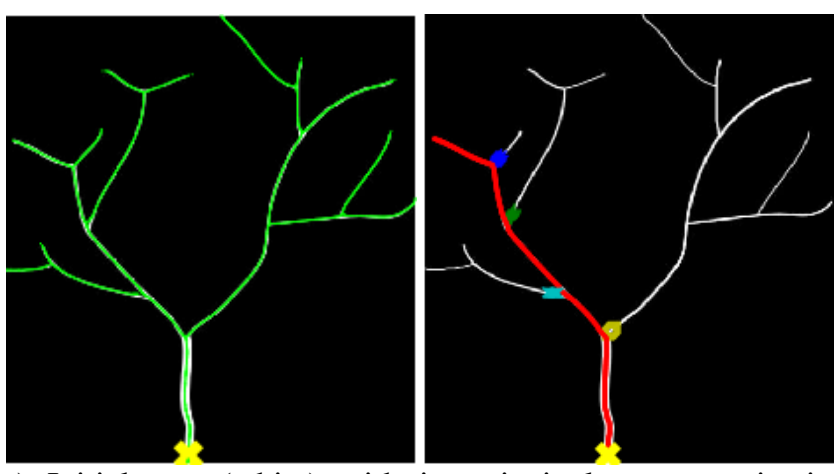

a) Initial tree (white) with its principal curve projection (green). b) Detection of nearby PC samples.

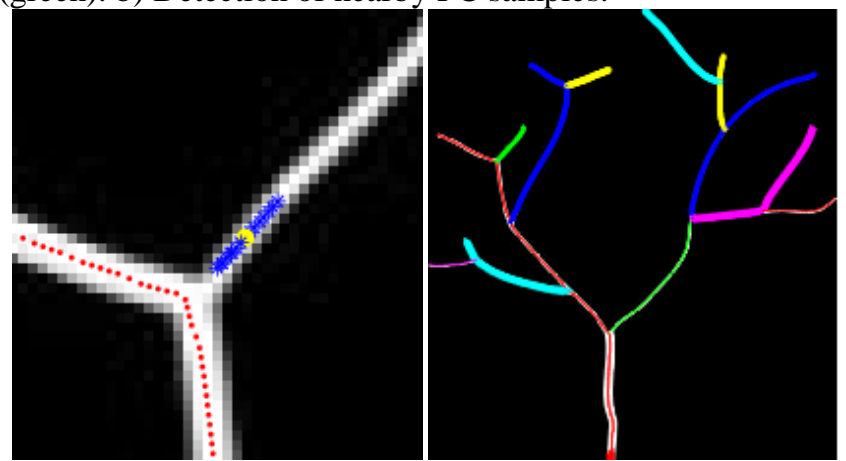

c) Zoomed look around a bifurcation d) Recursive Tracing result.

Fig. 1 Recursive tracing method.

envelop are in general elongated in distinct clusters and mainly originate from distinct principal curves as shown in Fig. 1(b). Fig. 1(c) shows the zoomed version of the top left branch, where blue stars represent nearby PC samples. Medioid-shift method [10] is used to estimate the modes of these clusters, and each mode is assumed as the bifurcation candidate, $\mathbf{s}_{\mathrm{i}}$. For every candidate, we shoot two trajectories in the direction given by $\mathbf{q}_{1}\left(\mathbf{s}_{\mathrm{i}}\right)$ and $-\mathbf{q}_{1}\left(\mathbf{s}_{\mathrm{i}}\right)$ using the tracing method defined before. If a trajectory coincides with the previously traced branch (at a junction sample), then branch is detected and the mode is simply connected to the junction sample, else we conclude that the tested trajectory diverges from the local principal curve. For the detected branches we start the tracing algorithm with $\mathbf{s}_{\mathrm{i}}$ as the seed location and the negative of the direction used for the test trajectory that converges to the previously traced branch. We continue to run the same recursion until no branch candidates remain. Fig.1(d) shows the final tracing result obtained by described recursions. Note that displayed iterations are the actual results of the proposed approach.

\section{RESULTS}

We first applied the proposed method to the synthetic data that is shown in Fig.1(a). The image size is $435 \times 500$ pixels and is generated by convolving the trace of the tree with a Gaussian kernel having size of $15 \times 15$ with increasing scale 


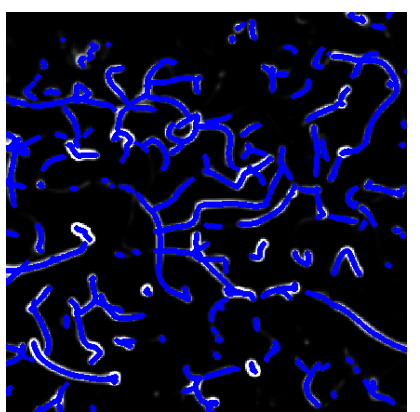

(a)

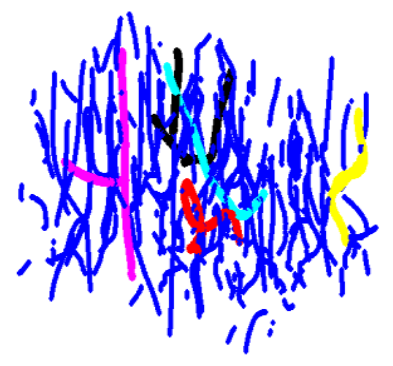

(c)

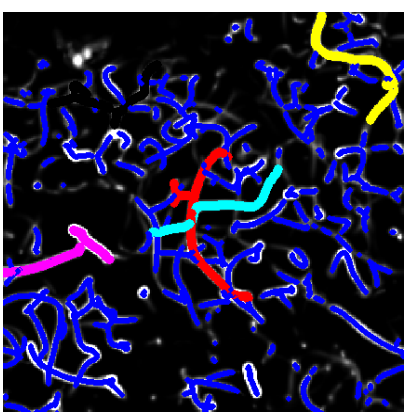

(b)

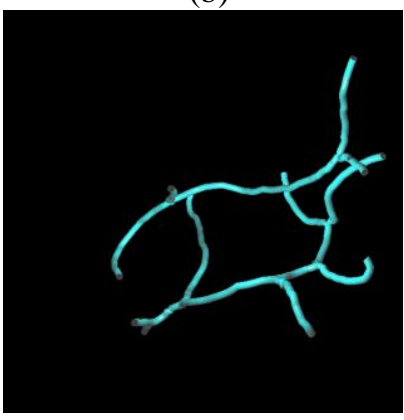

(d)
Fig. 2 (a) Projected samples 3D microscopy image stack. (b) Overlaid four vessel tracing. (c) 3D view of the projected samples and the tracings. (d) Final result: reconstructed vessel.

in y-direction (assuming the image origin is at upper-left corner). Mean centerline deviation between the algorithm result and original trace is calculated as 0.2058 pixels. Next, the proposed method is tested on two 3D image stacks obtained from multi photon microscopy. Each stack has 110 and 62 images, and the size of the images is $512 \times 512$ pixels as shown in Fig. 2(a). Between image stacks, there is an overlap in $\mathrm{z}$ direction that enables us to register stacks to each other. Here, principal curve projections are depicted with blue dots. Fig. 2(b-c) display four vessel trace in 2D and $3 \mathrm{D}$ respectively obtained using the proposed approach. Fig. 2(d) shows the 3D reconstruction of a sample in 3D.

\section{DISCUSSION}

In this paper, we described a tracing method for curvilinear structures. Proposed method utilizes Gaussian interpolation to define a twice differentiable function using variable kernel size bandwidths that is consistent with the local geometry. Unlike other methods, this method does not require segmentation as a pre-processing step. At each iteration, correction term pulls the trace to the underlying principal curve, which enables us to obtain accurate traces. Bifurcations are handled without any explicit effort, and recursive implementation provides a compact and simple solution for the challenging task. Future work will include quantitative analysis of results obtained from the proposed method when manually labeled data becomes available.
Acknowledgment: The authors would like to thank David Boas and his group for providing the image stacks used in this paper.

\section{REFERENCES}

[1] D. Lesagea, E.D. Angelinib, I. Blochb and G. FunkaLea, "A review of 3D vessel lumen segmentation techniques: Models, features and extraction schemes", Medical Image Analysis, vol. 13, no. 6, pp. 819-845, 2009.

[2] E. Sorantin, C. Halmai, B. Erdohelyi, K. Palagyi, L. Nyul, K. Olle, B. Geiger, F. Lindbichler, G. Friedrich, and K. Kiesler, "Spiral-ct-based assessment of tracheal stenoses using 3-dskeletonization", IEEE Trans. on Med. Img., vol. 21, pp. 263-273, March 2002.

[3] E. Bas and D. Erdogmus, "Piecewise Linear Cylinder Models For 3-Dimensional Axon Segmentation". 4th IEEE International Symposium on Biomedical Imaging: From Nano to Macro, 2010.

[4] O. Wink, W.J. Niessen and M.A. Viergever, "Multiscale Vessel Tracking", IEEE Transactions on Medical Imaging, vol. 23, no. 1, pp. 130-133, 2004.

[5] A.F. Frangi, W.J. Niessen, R.M. Hoogeveen, T. van Walsum and M.A. Viergever, "Model-based Quantitation of 3-D magnetic Resonance Angiographic

Images." IEEE Transactions on Medical Imaging, vol. 18, no. 10, pp. :946-956, 1999.

[6] E. Bas and D. Erdogmus, "Principal Curve Tracing", European Symposium on Artificial Neural Networks, 2010.

[7] H.E.Cetingul, G.Plank, N.Trayanova, and R.Vidal, "Stochastic Tractography in 3-D Images via Nonlinear Filtering and Spherical Clustering, Probabilistic Models for Medical Image Analysis", Workshop at MICCAI'09, London, UK, September 2009.

[8] G. González, E. Türetken, F. Fleuret and P. Fua, "Delineating Trees in 2D Images and 3D Image-Stacks", proceedings of the IEEE international conference on Computer Vision and Pattern Recognition (CVPR), 2010.

[9] U. Ozertem, "Locally Defined Principal Curves and Surfaces", PhD Dissertation, Oregon Health and Science University, Portland, OR, Aug 2008.

[10] Y.A. Sheikh, E.A. Khan and T. Kanade, " Modeseeking by medoidshifts", IEEE 11th International Conference on Computer Vision, pp.1-8, 2007. 